\title{
Inactivation of Tor proteins affects the dynamics of endocytic proteins in early stage of endocytosis
}

\author{
B Tenay \\ MSU Graduate Student \\ E Kimberlin \\ MSU Undergraduate \\ M Williams \\ MSU Undergraduate \\ D Juliette \\ MSU Undergraduate \\ J Fakilahyel \\ MSU Undergraduate
}

See next page for additional authors

Follow this and additional works at: https://bearworks.missouristate.edu/articles-cnas

\section{Recommended Citation}

Tenay, Brandon, Evin Kimberlin, Michelle Williams, Juliette Denise, Joshua Fakilahyel, and Kyoungtae Kim. "Inactivation of Tor proteins affects the dynamics of endocytic proteins in early stage of endocytosis." Journal of biosciences 38, no. 2 (2013): 351-361.

This article or document was made available through BearWorks, the institutional repository of Missouri State University. The work contained in it may be protected by copyright and require permission of the copyright holder for reuse or redistribution.

For more information, please contact BearWorks@library.missouristate.edu. 
Authors

B Tenay, E Kimberlin, M Williams, D Juliette, J Fakilahyel, and Kyoungtae Kim 


\title{
Inactivation of Tor proteins affects the dynamics of endocytic proteins in early stage of endocytosis
}

\author{
Brandon Tenay, Evin Kimberlin, Michelle Williams, Juliette Denise, Joshua Fakilahyel \\ and Kyoungtae Kim* \\ Department of Biology, Missouri State University, 901 South National Ave, Springfield, \\ MO 65897, USA \\ *Corresponding author (Fax, 417-836-5126; Email, kkim@missouristate.edu)
}

\begin{abstract}
Tor2 is an activator of the Rom2/Rho1 pathway that regulates $\alpha$-factor internalization. Since the recruitment of endocytic proteins such as actin-binding proteins and the amphiphysins precedes the internalization of $\alpha$-factor, we hypothesized that loss of Tor function leads to an alteration in the dynamics of the endocytic proteins. We report here that endocytic proteins, Abp1 and Rvs167, are less recruited to endocytic sites not only in tor 2 but also tor 1 mutants. Furthermore, we found that the endocytic proteins Rvs167 and Sj12 are completely mistargeted to the cytoplasm in tor $1 \Delta$ tor $2^{\text {ts }}$ double mutant cells. We also demonstrate here that the efficiency of endocytic internalization or scission in all tor mutants was drastically decreased. In agreement with the Sj12 mislocalization, we found that in tor $1 \Delta$ tor $2^{\text {ts }}$ double mutant cells, as well as other tor mutant cells, the overall $\mathrm{PIP}_{2}$ level was dramatically increased. Finally, the cell wall chitin content in tor $2^{\text {ts }}$ and tor $1 \Delta$ tor $2 t s$ mutant cells was also significantly increased. Taken together, both functional Tor proteins, Tor1 and Tor2, are essentially required for proper endocytic protein dynamics at the early stage of endocytosis.
\end{abstract}

[Tenay B, Kimberlin E, Williams M, Denise J, Fakilahyel J and Kim K 2013 Inactivation of Tor proteins affects the dynamics of endocytic proteins in early stage of endocytosis. J. Biosci. 38 351-361] DOI 10.1007/s12038-013-9326-7

\section{Introduction}

Target of rapamycin (TOR) proteins are found across eukaryotic organisms, including mammals and yeast. In mammals, it is known as mTOR (mechanistic TOR) and forms two complexes: mTOR complex 1 (mTORC1) and mTOR complex 2 (mTORC2) (Hall 2008; Laplante and Sabatini 2012). mTOR functions by modulating the activities of a diverse array of downstream effector proteins to control many specific signaling events within the cell (Kim et al. 2002; Loewith et al. 2002; Manning and Cantley 2007). In particular, it has been shown that mTORC1 is involved in controlling biosynthesis of proteins and growth (Laplante and Sabatini 2012), while mTORC2 plays a role in regulating cellular processes including cytoskeletal organization (Jacinto et al. 2004).
In S. cerevisiae, there are two known Tor proteins, Tor1 and Tor2, which were identified while screening mutants that showed resistance to rapamycin (Cafferkey et al. 1993). Tor proteins share a high homology (67\%) at the nucleotide level (Helliwell et al. 1994). There are two distinct Tor complexes: TORC1 and TORC2. TORC1 is composed of Tor1 (or Tor2), Kog1 and Lst8, while TORC2 contains Tor2, Avo1, Avo2, Avo3, Bit61 and Lst8 (Loewith et al. 2002; Wedaman et al. 2003). Tor1 is a PIK-related protein kinase and, similar to its mammalian counterpart mTor, regulates the synthesis of proteins, which is required for the cell cycle progression from $\mathrm{G} 1$ to $\mathrm{S}$ phase (Barbet et al. 1996). Although it has been known that Tor1 and Tor2 share many biological functions, a unique feature of Tor2 similar to mTORC2 is that Tor2 regulates the reorganization of the actin cytoskeleton (Schmidt et al. 1996).

Keywords. Abp1; endocytosis; $\mathrm{PIP}_{2}$; scission; Tor2

Supplementary materials pertaining to this article are available on the Journal of Biosciences Website at http://www.ias.ac.in/jbiosci/ jun2013/supp/tenay.pdf 
Consistent with the functional divergence between the two kinases, their subcellular locations were found to be different from each other: Tor1 at the vacuole and Tor2 at the plasma membrane (Aronova et al. 2007; Berchtold and Walther 2009; Cardenas and Heitman 1995; Sturgill et al. 2008).

It is known that the actin cytoskeleton is required for the proper function of endocytosis in yeast (Kaksonen et al. 2005). Once an endocytic site has been selected, actin-independent endocytic proteins, including clathrin, are recruited first, which is followed by the recruitment of actin and actin-binding proteins, Abp1 and Arp2/3 complex (Kaksonen et al. 2003, 2005; Newpher et al. 2005). Abp1 and Arp2/3 complex stimulate actin polymerization, which facilitates the invagination of the plasma membrane (Kaksonen et al. 2005). Scission of the invagination takes place at the neck of the invagination with the help of Rvs161, Rvs167 and Vps1 (Idrissi et al. 2008; Kaksonen et al. 2005; Smaczynska-de et al. 2012). During and after scission of the endocytic vesicle, it appears that $\mathrm{Sj} 12$ plays a role for the proper uncoating of endocytic proteins from the vesicle (Toret et al. 2008). With the known functional significance of the actin cytoskeleton in endocytosis, it has been suggested that the severity of actin defect correlates tightly with the severity of endocytic defect (Engqvist-Goldstein and Drubin 2003). For instance, cells containing mutant alleles of TOR2 gene exhibited depolarization of the actin cytoskeleton and defects in their ability to internalize $\alpha$-factor pheromone via receptor-mediated endocytosis (deHart et al. 2003; Schmidt et al. 1996). However, the specific roles of Tor2 in the dynamics of endocytic proteins during early receptor-mediated endocytosis have not yet been documented. Recently, research has focused on quantitating the effects of gene mutations on the dynamics of endocytic proteins at the site of receptor-mediated endocytosis. In particular, with the help of real-time live-cell imaging of GFP-fusion proteins, researchers are able to quantitate the dynamic recruitment and internalization events of endocytic proteins, occurring at the endocytic sites. Using a similar approach we investigated effects of partial and complete loss of Tor function on the dynamics of Abp1-GFP, Rvs167-GFP and Sj12-GFP, and here present that intact Tor proteins are required both for proper dynamics of these endocytic proteins and efficient internalization event.

\section{Materials and methods}

\subsection{Yeast strain construction and media}

Yeast strains used in this study are listed in table 1. Strains expressing GFP-fused proteins were constructed by integrating respective GFP sequence at the $3^{\prime}$ end of the gene of interest as described previously (Longtine et al. 1998; Kim et al. 2006; Nannapaneni et al. 2010). Mutant strains tor $1 \Delta$ in which TOR1 locus is replaced by a LEU2 cassette (Helliwell et al. 1994), $\operatorname{tor}^{2 s}$ (a Tor2 PI kinase temperature-sensitive mutant harbouring the tor ${ }^{\text {kin- }}$ allele) (Schmidt et al. 1996), tor $1 \Delta$ tor $2^{t s}$ and Tor2 overexpression (Schmidt et al. 1996) were obtained from Michael Hall's lab. Since TOR2 is an essential gene, Kunz et al. (1993) replaced the TOR2 with a $A D E 2$ and introduced a plasmid with a mutation in the kinase domain of Tor2 to produce tor ${ }^{t s}$ strain. Tor2 overexpression strain (Schmidt et al. 1996) was constructed to overexpress Tor 2 under the control of a GAL-promoter, and $t o r 2^{\text {ts }}$ cells overexpressing Tor 2 were able to grow even at the restricted temperature. Tor 2 overexpression strain was gift from Dr. Michael Hall (University of Basel). All yeast strains except Tor 2 overexpressing cells were grown in yeast peptone dextrose (YPD) and cultured at either $30^{\circ} \mathrm{C}$ or $38^{\circ} \mathrm{C}$. Tor2 overexpressing cells were grown in SD-URA-mediacontaining galactose $(2 \%)$ in order to induce overexpression of Tor2. Cells expressing a $\mathrm{PIP}_{2}$-binding GFP-2XPH domain were constructed by introducing the GFP-2XPH PLC $\delta$ plasmid (Kindly provided by Scott Emr, Cornell University) by following one-step transformation protocol (Chen et al. 1992).

\subsection{Spinning confocal microscopy}

Time-lapse movies and still images of GFP labeled cells were made with a spinning disk confocal microscope. The system included an inverted Olympus 1X81 microscope, a Yokogawa CSUX1 spinning disk head, a 100× numerical aperture (NA) 1.4 PlanApo oil objective, and an electron amplified CCD (ImageEM, Hamamatsu). The temperature at the stage was maintained at $30^{\circ} \mathrm{C}$ or $38^{\circ} \mathrm{C}$. Images were focused at an equatorial plane of the cells. Time-lapse movies of GFP labeled proteins were captured at 2 frames/ $\mathrm{s}$ for movie duration time of $1 \mathrm{~min}$. $\mathrm{PIP}_{2}$ was labeled by expressing GFP-2xPH (PLC $\delta$ ) as previously described (Stefan et al. 2002). To quantitate $\mathrm{PIP}_{2}$ expression levels, 13 small-budded cells from each strain expressing GFP$2 \mathrm{xPH}(\mathrm{PLC} \delta$ ) were randomly chosen to determine the average total GPF fluorescence per cell. To visualize yeast cell wall, cells were stained with calcofluor as previously described (Bickle et al. 1998).

\subsection{Measurement of patch lifetime at the membrane and kymograph}

Patch lifetime at the membrane was determined by calculating the time spent by a GFP-fused protein at the membrane (from the time the patch appeared to the time at which the patch was internalized from its origin or disappeared), as previously described (Murphy et al. 2011). Mean patch lifespan of GFP-fused proteins at the membrane was determined by averaging lifespan of the florescences from more 
Table 1. Yeast strains used for this study

\begin{tabular}{|c|c|c|}
\hline Strain & Genotype & Source \\
\hline KKY 917 & Mata leu2-3,112 ura3-52 rme1 trp1 his4 GAL+ HMLa torl::LEU $U_{2-4}$ & (Schmidt et al. 1996) \\
\hline KKY 918 & Mata leu2-3,112 ura3-52 rme1 trp1 his4 GAL+ HMLa ade2 tor $2:: A D E_{2-3} / \mathrm{YCplac}_{111:: t_{2-21}}{ }^{\text {ts }}$ & (Schmidt et al. 1996) \\
\hline KKY 919 & $\begin{array}{l}\text { Mata leu2-3,112 ura3-52 rmel trp1 his4 GAL+ HMLa ade2 torl::HIS } S_{3-3} \text { tor } 2:: A D E_{2-3} / \\
\text { YCplac111::tor2-21 }\end{array}$ & (Schmidt et al. 1996) \\
\hline KKY 920 & $\begin{array}{l}\text { Mata leu2-3,112 ura3-52 rme1 trp1 his4 GAL+ HMLa ade2 tor2::ADE } E_{2-3} / p J K 5 \\
\text { (pSEYC68galp::TOR2) }\end{array}$ & (Schmidt et al. 1996) \\
\hline KKY 0051 & Mata his3 leu2 ura3, ABP1-GFP-HIS3 & Invitrogen \\
\hline KKY 0960 & Mata leu2-3,112 ura3-52 rme1 trp1 his4 GAL+ HMLa tor $: \because L E U_{2-4}, A B P 1-G F P-T R P 1$ & This study \\
\hline KKY 0961 & $\begin{array}{l}\text { Mata leu2-3,112 ura3-52 rme1 trp1 his4 GAL+ HMLa ade2 tor } 2:: A D E_{2-3} / \mathrm{YCplac}_{111: \text { tor } 2-21}{ }^{\text {ts }} \text {, } \\
\text { ABP1-GFP-TRP1 }\end{array}$ & This study \\
\hline KKY 0962 & $\begin{array}{l}\text { Mata leu2-3,112 ura3-52 rme1 trp1 his4 GAL+ HMLa ade2 torl::HIS } S_{3-3} \text { tor } 2: \because A D E_{2-3} / \\
\text { YCplac111::tor2-21 }\end{array}$ & This study \\
\hline KKY 0963 & $\begin{array}{l}\text { Mata leu2-3,112 ura3-52 rme1 trp1 his4 GAL + HMLa ade2 tor2::ADE } E_{2-3} / p J K 5 \\
\text { (pSEYC68galp::TOR2), ABP1-GFP-TRP1 }\end{array}$ & This study \\
\hline KKY 0661 & Mata his3 leu2 lys2 ura3, RVS167-GFP-HIS & This study \\
\hline KKY 1009 & Mata leu2-3,112 ura3-52 rme1 trp1 his4 GAL+ HMLa tor $1: L E U_{2-4}, R V S 167-G F P-T R P 1$ & This study \\
\hline KKY 1010 & $\begin{array}{l}\text { Mata leu2-3,112 ura3-52 rme1 trp1 his4 GAL+ HMLa ade2 tor } 2:: A D E_{2-3} / \mathrm{YCplac}_{111: \text { tor2-21 }}{ }^{\text {ts }} \text {, } \\
\text { RVS167-GFP-TRP1 }\end{array}$ & This study \\
\hline KKY 1011 & $\begin{array}{l}\text { Mata leu2-3,112 ura3-52 rme1 trp1 his4 GAL+ HMLa ade2 tor } 1: H I S_{3-3} \text { tor } 2: \because A D E_{2-3} / \\
\text { YCplac111:tor2-21 }{ }^{\prime} \text {, RVS167-GFP-TRP1 }\end{array}$ & This study \\
\hline KKY 455 & Mata his3 leu2 met15 ura3, SJL2-GFP-HisMx6 & This study \\
\hline KKY 1014 & 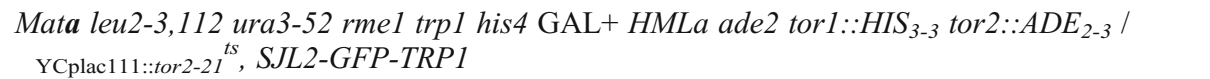 & This study \\
\hline KKY 1075 & 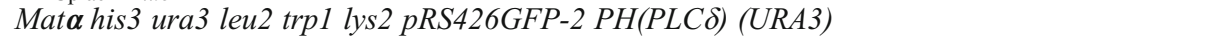 & This study \\
\hline KKY 1077 & $\begin{array}{l}\text { Mata leu2-3,112 ura3-52 rmel trp1 his4 GAL+ HMLa tor } 1: L E U_{2-4}, p R S 426 G F P-2 \\
\text { PH(PLC }) \text { (URA3) }\end{array}$ & This study \\
\hline KKY 1079 & 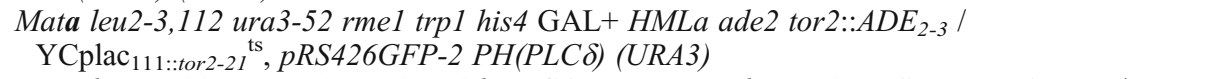 & This study \\
\hline KKY 1081 & 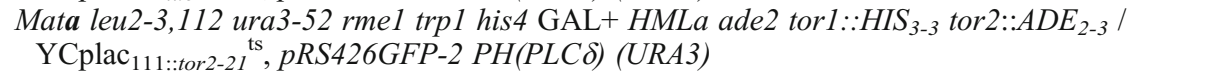 & This study \\
\hline
\end{tabular}

than at least 20 patches. Patches that at any time appeared too close to another patch were excluded from our analysis. In order to directly visualize the duration and fluorescence intensity of the patch on the membrane, a kymographic representation of GFP-fused proteins in a single patch over time was made using Slidebook (v.5). Average fluorescence intensity of the GFP-patches was determined using Image J (v.1.4).

\section{Results}

\subsection{Partial and complete loss of Tor function affects Abpl dynamics}

It has been previously described that the rate of $\alpha$-factor internalization by receptor-mediated endocytosis (RME) was attenuated in tor $2^{G 2128 R}$ kinase mutant cells that express arginine instead of glycine at the 2128th residue within the Tor2 protein (deHart et al. 2003). In order to investigate how Tor2 plays a role in both the recruitment dynamics of endocytic proteins and the scission efficiency during RME, a well-characterized RME marker Abp1 was fused with GFP and the dynamic behavior of Abp1-GFP in cells was analysed at both $30^{\circ} \mathrm{C}$ and $38^{\circ} \mathrm{C}$, after recording time-lapse fluorescence images. Actin-binding protein Abp 1 arrives at endocytic sites approximately 5-8 s before scission event, moves in a directed manner away from the endocytic site, and dissociates from the post-internalized endocytic patch to be reused at future endocytic sites (Kim et al. 2006; Toret et al. 2008). Representative fluorescent images of Abp1-GFP patches in $\mathrm{Wt}$ and tor mutant cells $\left(\right.$ tor $1 \Delta$, tor $2^{\text {ts }}$, tor $1 \Delta$ tor $2^{\text {ts }}$ and tor2 overexpressing) are shown in figure $1 \mathrm{~A}$. We found that the mean membrane lifespans of Abp1-GFP in all tor mutant cells except Tor2 over expressing cells were similar to that of $\mathrm{Wt}$ cells at both $30^{\circ} \mathrm{C}$ and $38^{\circ} \mathrm{C}(8.2 \pm 2.2 \mathrm{~s})$ (figure $1 \mathrm{~B}$ and $\mathrm{C}$ ). These results suggest that Tor1 and functional Tor2-kinase domain are not required for both the targeting of Abp1-GFP to endocytic sites and its subsequent maturation event. However, overexpression of Tor2 led to a robust increase in Abp1-GFP lifespan ( 30 s), more than 3fold when compared with $\mathrm{Wt}$, indicating that the overexpression negatively affects the maturation event of 

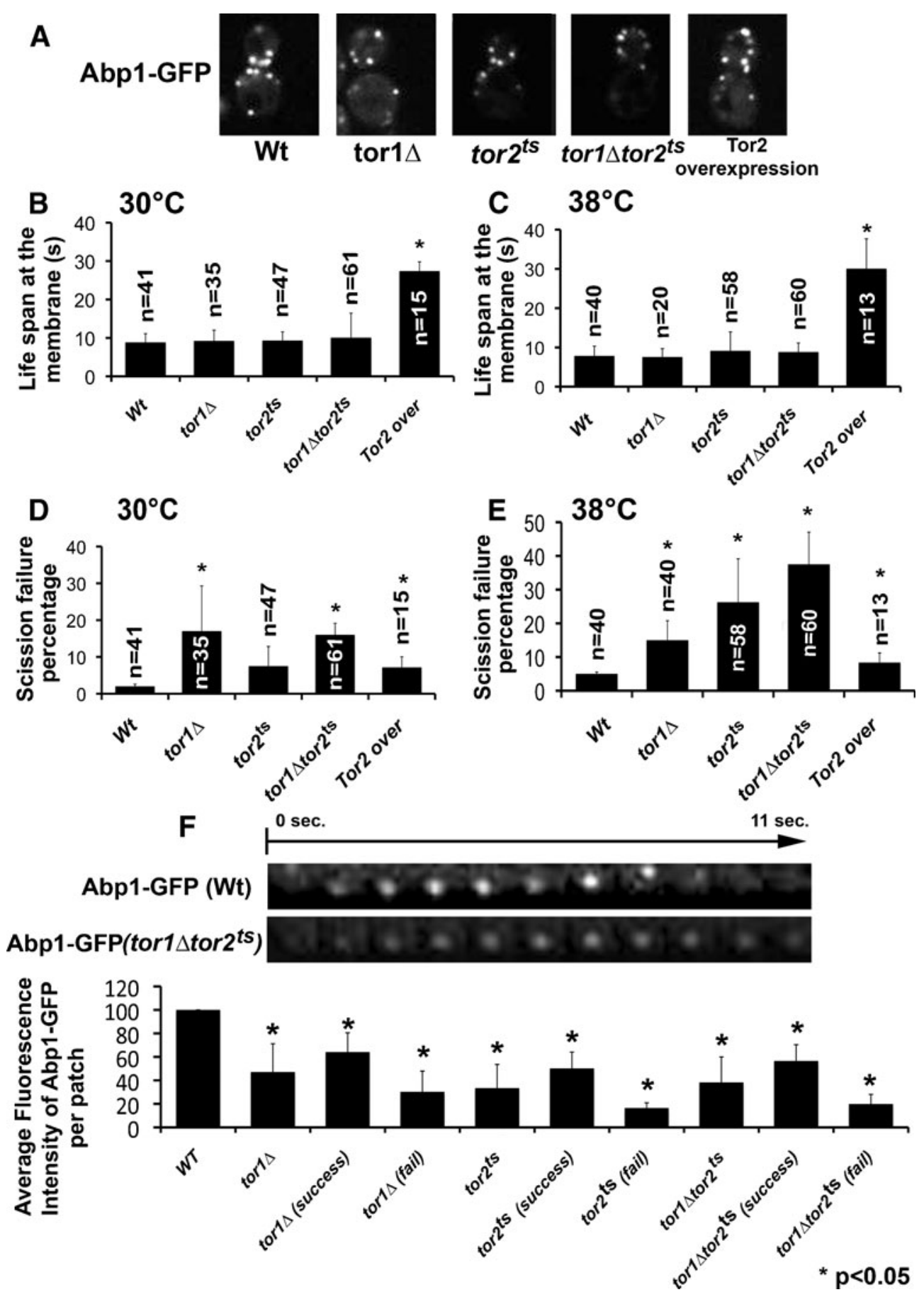

Figure 1. Partial or complete inactivation of Tor proteins is accompanied by defects in endocytic internalization and relatively low amount of Abp1-GFP at endocytic sites. (A) Representative images of Abp1-GFP in Wt (KKY 0051), tor1 $\Delta$ (KKY 0960), tor $2^{\text {ts }}$ (KKY 0961), tor1 $\Delta$ tor $2^{\text {ts }}$ (KKY 0962), and Tor2 overexpression (KKY 0963) cells. (B and C) Mean membrane lifespan of Abp1-GFP at $30^{\circ} \mathrm{C}$ and $38^{\circ} \mathrm{C}$. Abp1-GFP patches were analysed to determine the mean membrane lifespan as described in the methods section $(\mathrm{n}=$ number of patches). (D and $\mathbf{E})$ Percent internalization or scission failure of Abpl-GFP patches at $30^{\circ} \mathrm{C}$ and $38^{\circ} \mathrm{C}$. Patches were counted as internalized if they moved away from the site of recruitment. Patches that failed to show internalization were counted as unsuccessful patches in scission. Scission efficiency decreases in all tested tor mutant cells. (F) Kymograph representation of Abp1-GFP patch at the cell membrane over time in Wt and tor $1 \Delta$ tor $2^{t s}$ cells. As shown in the kymograph, lifespans of both patches are similar, but the patch in tor $1 \Delta$ tor $2^{\text {ts }}$ cell is dimmer. Fluorescence intensity of ten to twenty Abp1GFP patches was analysed to get the mean fluorescence intensity. The mean fluorescence intensity of Abp1-GFP in Wt cells was normalized to $100 \%$. An asterisk $(*)$ indicates a statistically significant difference $(\mathrm{p}<0.05)$ as compared to the mean fluorescence intensity of Wt Abp1-GFP patch. 
Abp1-GFP. After recruited to endocytic sites, endocytic proteins that are physically associated with the endocytic vesicles are internalized. We, therefore, determined the extent to which Abp1-GFP patches undergo successful internalization or scission in tor mutant cells. Abp1-GFP patches that fail to move away from the site of recruitment were considered to be 'scission failure patches'. In most cases, Abp1-GFP appears to be dissociated from patches that fail to internalize, thereby those can also be considered as aborted patches. Thus, we concluded that the low rate of scission success in the tor mutants is attributed to abortion in endocytosis that might be caused by premature disassembly of Abp 1 . Strikingly, scission failure increased 4- to 9-fold at both $30^{\circ} \mathrm{C}$ and $38^{\circ} \mathrm{C}$ in tor mutant cells, with the highest failure rate in tor $1 \Delta$ tor $2^{\text {ts }}$ cells ( $40 \%$ ) (figure $1 \mathrm{C}$ and D). In addition to the low efficiency in endocytic scission, the fluorescence intensity of Abp1-GFP patches in tor mutants was dimmer than the patches observed in Wt cells, manifested by Kymographic representation shown in figure $1 \mathrm{~F}$ and by a colocalization assay shown in supplementary figure $1 \mathrm{~A}$. We thus further quantitated average fluorescence intensity of Abp1-GFP per patch and found that the extent of Abp1GFP fluorescence intensity in tor mutant cells decreased significantly (figure 1F). In particular, the fluorescence intensity of scission failure patches in tor mutant cells was far lower than that of a successfully internalizing Abp1-GFP patch in the same mutant cells (figure 1F). These results suggest that less Abp1-GFP is recruited to the endocytic sites in tor mutant cells than Wt. All together, we came to the conclusion that loss of Tor protein activities affects receptor-mediated endocytosis by significantly decreasing endocytic scission efficiency, which may be partially attributable to a suboptimal recruitment of Abp1 to the endocytic sites.

\subsection{Impaired membrane targeting of Rvs167 in tor mutant cells}

Upon observing the decrease in scission efficiency of Abp1GFP in tor mutant cells both at 30 and $38^{\circ} \mathrm{C}$, we set out to examine whether or not Tor proteins affect the dynamics of endocytic protein Rvs167, since Rvs167 has been shown to help membrane invagination and scission events (Kaksonen et al. 2005). Consistent with previous findings (Balguerie et al. 1999; Kaksonen et al. 2005), Rvs167-GFP in Wt cells was localized to cortical membrane patches. It was also observed that Rvs167-GFP, in tor $1 \Delta$ and tor $2^{\text {ts }}$ mutant cells, is recruited to the cortical endocytic sites (figure 2A). However, it appears that the number of Rvs167-GFP patch recruited to the cortex was decreased (see below and supplementary figure 1B). However, surprisingly, we found that in tor $1 \Delta$ tor $2^{t s}$ mutant cells, Rvs167-GFP was completely mislocalized to the cytoplasm as punctate structures (figure $2 \mathrm{~A}$ and supplementary figure 1B). Often we observed patches that appeared associated to the cell cortex in the double mutant, but no patches were formed de novo at the cortex. The average membrane lifespans of Rvs167 in all tor mutant and $\mathrm{Wt}$ cells were similar to each other at $30^{\circ} \mathrm{C}$ (figure $2 \mathrm{C}$ ). Although the membrane lifespans in tor $1 \Delta$ and $t o r 2^{t s}$ cells were slightly decreased at $38^{\circ} \mathrm{C}$, the time difference was not statistically significant. The extent to which Rvs167-GFP patches undergo successful internalization or scission in tor mutant cells was determined as described above. At both $30^{\circ} \mathrm{C}$ and $38^{\circ} \mathrm{C}$, the averaged rate of scission failure was drastically increased both in tor $1 \Delta$ and tor $^{\text {ts }}$ cells (figure $2 \mathrm{D}$ and $\mathrm{E}), 2$-fold $\left(\sim 20 \%\right.$ at $\left.30^{\circ} \mathrm{C}\right)$ and 4 -fold ( $36 \%$ in $\operatorname{tor}^{\text {ts }}$ at $38^{\circ} \mathrm{C}$ ), respectively, when compared to that of $\mathrm{Wt}(8.9 \%)$. However, the difference in scission rate between those cells was not statistically significant at $30^{\circ} \mathrm{C}$, due to a high standard deviation in torls and tor $2^{t s}$. The experimental results in figure $2 \mathrm{E}$ show that at $38^{\circ} \mathrm{C}$ the drastic difference in averaged scission rate between $\mathrm{Wt}$ and mutant strains is supported by a statistical analysis, as manifested by $p$-values being $<0.05$. Next, we quantified the number of Rvs167-GFP patches formed at the membrane cortex of a cell within a minute. Our analysis showed that the average numbers of Rvs167-GFP patches at the membrane both in tor $1 \Delta$ and $\operatorname{tor} 2^{t s}$ cells were $\sim 5$, thus $25 \%$ of that of Wt $(20 \pm 2.3$ patches/min) (figure $2 \mathrm{~F}$ and $\mathrm{G})$. Kymograph representations of Rvs167-GFP patches in tor mutant cells showed a significant decrease in the fluorescence intensity of Rvs167-GFP (figure 2H). The quantification of Rvs167-GFP fluorescence intensity revealed that there was significantly less Rvs167-GFP being recruited to the endocytic sites in tor mutant cells when compared to Wt (figure 2I).

\subsection{Sjl2 mislocalization in tor $1 \Delta$ tor $2^{\text {ts }}$ mutant cells had a significant effect on membrane $\mathrm{PIP}_{2}$ levels}

Because Abp1 is known to be an important factor for the recruitment of Sj12 to endocytic sites (Stefan et al. 2002, 2005), we examined Sj12 localization in tor mutant cells that show defects in the proper dynamics of Abp1 and Rvs167 (figures 1 and 2). $\mathrm{Sj} 12$ has been implicated in endocytosis via optimization of the plasma membrane PIP $_{2}$ levels (Stefan et al. 2002). As shown in figure 3, Sjl2-GFP was properly targeted to membrane cortex in Wt. In order to visualize Sj12-GFP in tor $1 \Delta$ and tor $^{\text {ts }}$ cells, we introduced the GFP sequence at the $3^{\prime}$ end of $S J L 2$, but found that all colonies grown on selective media were found to be false-positives according to our analytical colony PCR (data not shown). Expression of Sj12-GFP in tor $1 \Delta$ tor $2^{\text {ts }}$ mutant cells was successful, and in these, Sj12-GFP was exclusively mistargeted to the cytoplasm, where it was seen as punctate patches (figure 3A and supplementary figure 1C). We also studied the localization of another synaptojanin-like protein, 


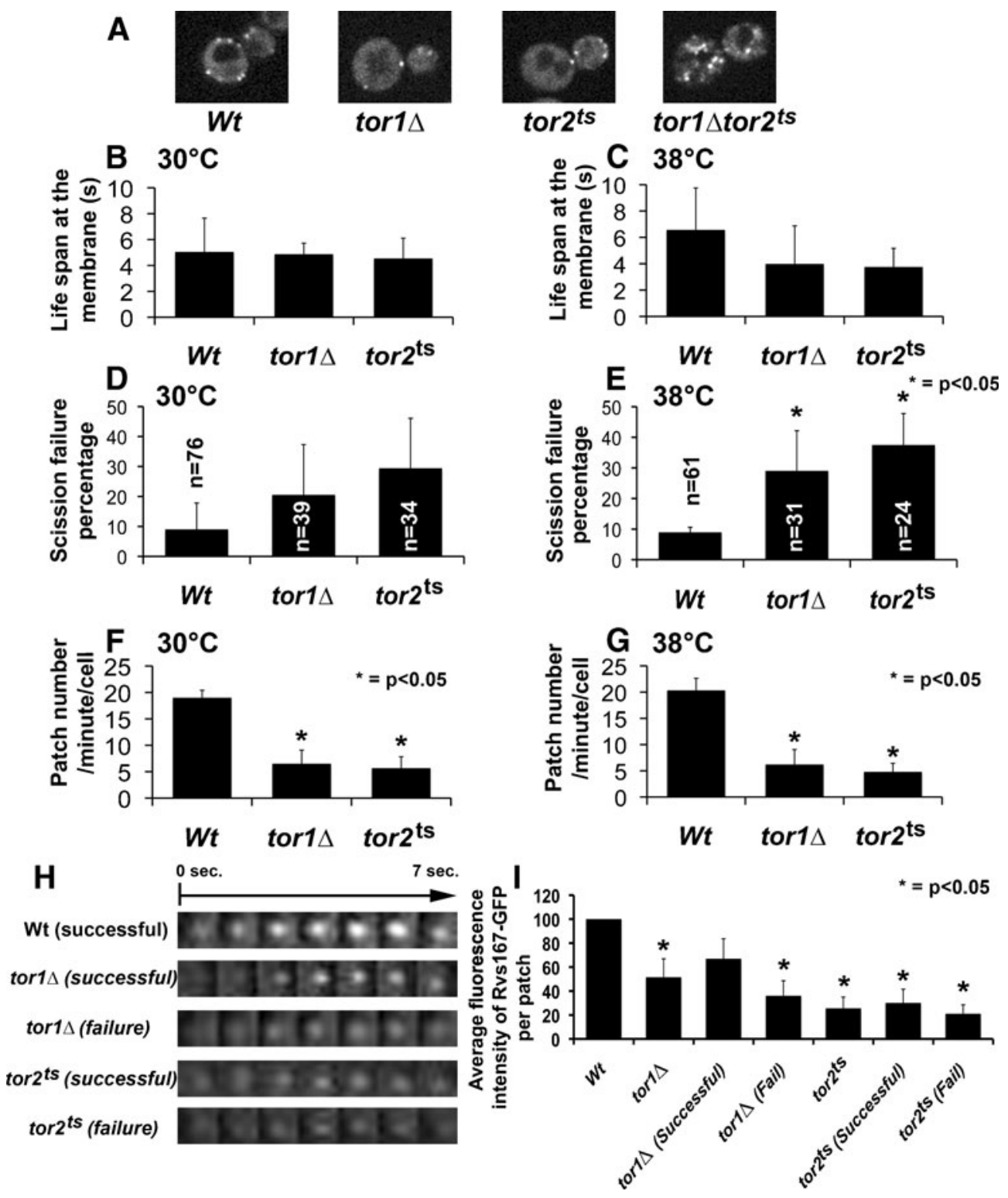

Figure 2. Internalization defect of Rvs167-GFP and a significant reduction of Rvs167-GFP fluorescence intensity in tor mutant cells. (A) Representative images of Rvs167-GFP in Wt (KKY 0061), torl $\Delta$ (KKY 1009), tor ${ }^{\text {ts }}$ (KKY 1010), and torl $\Delta$ tor $2^{t s}$ (KKY 1011). (B and C) Mean membrane lifespan of Rvs167-GFP patches at $30^{\circ} \mathrm{C}$ and $38^{\circ} \mathrm{C} .20$ to 70 Rvs167-GFP patches were analysed to determine the mean membrane lifespan as described in the methods section. No significant defect in membrane lifespan was observed. (D and E) Percent scission failure of Rvs167-GFP patches at $30^{\circ} \mathrm{C}$ and $38^{\circ} \mathrm{C}$. Scission efficiency decreases in all tor mutant cells. An asterisk $(*)$ indicates a statistically significant difference $(\mathrm{p}<0.05)$ as compared to the mean scission failure percentage of Wt Rvs167-GFP patch. (F and $\mathbf{G})$ Mean number of Rvs167-GFP patches that form at the plasma membrane during 1 min movie duration. 20 to 70 Rvs167-GFP patches were analysed to determine the mean number of membrane patches that formed. In tor mutant cells, a significant reduction in number of Rvs167GFP patches when compared to that in Wt $(*$ indicating $\mathrm{p}<0.05)$. (H) Kymograph representation of Rvs167-GFP patch at the cell membrane over time in $\mathrm{Wt}$, tor $1 \Delta$, and $\operatorname{tor}^{t s}$. (I) The mean fluorescence intensity of Rvs167-GFP patches $(\mathrm{n}=20-70)$. An asterisk $(*)$ indicates a statistically significant difference $(\mathrm{p}<0.05)$ as compared to the mean fluorescence intensity of Wt Rvs167-GFP patch.

Sj11, in the tor double mutant, and found a similar abnormal Sj11 localization in the cytoplasm (data not shown). It was shown that synaptojanins deficient cells (for example, sjll $\Delta$ sjl2 $\Delta$ double mutant) exhibit a robust increase in the level of membrane $\mathrm{PIP}_{2}$ (Stefan et al. 2002). Based on our finding of the severe mislocalization of Sj11 and Sj12 in the tor double mutant cells, we predicted that the double mutant cells would express an increased level of membrane $\mathrm{PIP}_{2}$, 
A

Wt tor1 $1 \Delta t o r 2^{\text {ts }}$

SjI2-GFP
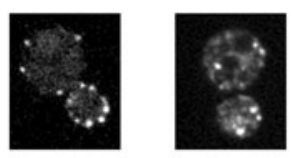

B

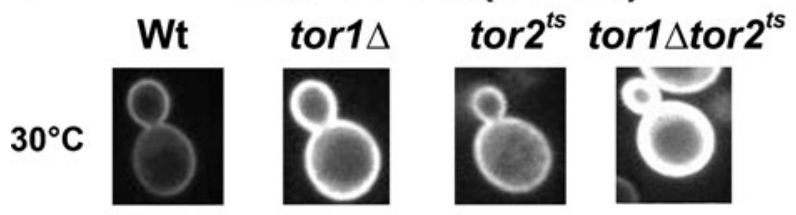

C

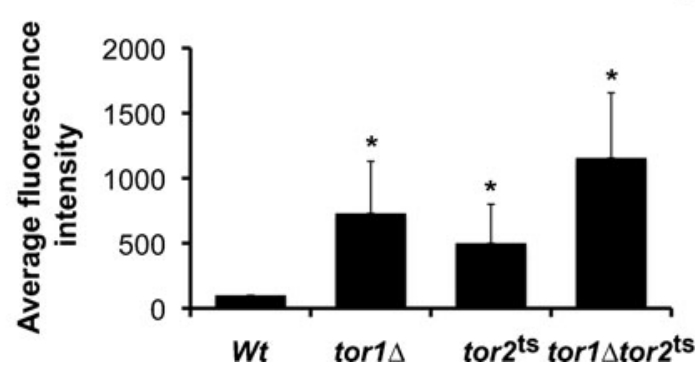

Figure 3. Mistargeting of Sj12 and hyperaccumulation of $\mathrm{PIP}_{2}$ in tor mutant cells. (A) Representative images of Sj12-GFP in $\mathrm{Wt}$ (KKY 455) and tor $1 \Delta$ tor $2^{\text {ts }}$ (KKY 1014) mutant cells. Noticeable cytoplasmic mistargeting of Sj12-GFP in tor $1 \Delta$ tor $2^{\text {ts }}$ was observed. (B) Representative images of membrane $\mathrm{PIP}_{2}$. In order to visualize $\mathrm{PIP}_{2}$, the GFP-2xPH (PLC 8 ) plasmid was expressed in Wt (KKY $1075)$, torl $\Delta$ (KKY 1077), tor $2^{\text {ts }}$ (KKY 1079), and tor $1 \Delta$ tor $2^{\text {ts }}$ (KKY 1081) cells. (C) The fluorescence intensity of PH-GFP for each bar was quantified in 13 randomly selected cells. The mean fluorescence intensity of PH-GFP in Wt cells was normalized to $100 \%$. An asterisk $(*)$ indicates a statistically significant difference $(\mathrm{p}<0.05)$ as compared to the mean fluorescence intensity of Wt.

and found that this was the case; approximately a 11-fold increase in the fluorescence levels of GFP fused pleckstrin homology $(\mathrm{PH})$ domain of $\mathrm{PLC} \delta$, a fluorescent reporter that binds $\mathrm{PIP}_{2}$, in the double mutant cells as shown in figure $3 \mathrm{~B}$ and $C$. In addition, we found the average GFP fluorescence intensity from tor $1 \Delta$ and tor $2^{t s}$ cells was significantly increased.

\subsection{Significant chitin accumulation in tor $2^{\text {ts }}$ and tor $1 \Delta$ tor $2^{\text {ts }}$ mutant cells}

Given that an endocytic blockade leads to an accumulation of cell wall chitin (Reyes et al. 2007), we examined cell wall chitin levels, using calcofluor white, in tor mutant cells. Representative images of calcofluor stained cell are shown in figure 4A. No change in the fluorescent intensity of calcofluor stained tor $1 \Delta$ cells was observed when compared to that of Wt cells, while chitin levels in $\operatorname{tor} 2^{t s}$ and tor $1 \Delta$ tor $2^{\text {ts }}$ even in a permissive condition (at $30^{\circ} \mathrm{C}$ ) were increased by $32 \%(\mathrm{p}=0.056)$ and $94 \%(\mathrm{p}<0.0001)$, respectively (figure 4B). In the non-permissive condition (at $38^{\circ} \mathrm{C}$ ), both $\operatorname{tor} 2^{\text {ts }}$ and tor $1 \Delta$ tor $2^{\text {ts }}$ mutant cells, as well as tor $1 \Delta$ cells, exhibited significantly increased levels of chitin.

\section{Discussion}

Results from our study provide the first evidence that Tor proteins, Tor1 and Tor2, are implicated in the proper recruitment of certain endocytic proteins and the subsequent scission efficiency of endocytic vesicles based on the following observations. First, upon partial and complete loss of Tor activity, Abp1 appears to arrive at endocytic sites normally, but scission efficiency was decreased (figure 1D and E), which could be partly attributed to less recruitment of Abp1 to the sites (figure 1F). Second, we observed that in all tor mutants, including tor $1 \Delta$, tor $2^{\text {ts }}$, and tor $1 \Delta$ tor $2^{\text {ts }}$ double mutants, amphiphysin Rvs167 recruitment to the endocytic site was affected, manifested by a drastic reduction $(\sim 75 \%)$ in the number of de novo endocytic site formations (figure $2 \mathrm{~F}$ and $\mathrm{G}$ ), as well as lower levels of Rvs167GFP fluorescence intensity at the site (figure $2 \mathrm{H}$ and I). In addition, the phosphoinositide phosphatase Sj12 was exclusively mistargeted to the cytoplasmic patch-like puncta in tor $1 \Delta$ tor $2^{\text {ts }}$ mutant cells (figure 3 ). Third, we concluded that the endocytic defects we observed are most likely due mainly to downregulation of Rom2 pathway via partial or complete Tor2 inactivation in our tor 2 mutant cells (tor $2^{\text {ts }}$ and tor $1 \Delta$ tor $\left.2^{t s}\right)$, which also leads to an increase in cell wall chitin level (figure 4).

\subsection{Less scission efficiency of endocytic vesicles carrying Abp1-GFP in tor mutants}

Abp1, a late marker for receptor-mediated endocytosis, is recruited to endocytic sites after the early endocytic adaptor Sla1 has been recruited. Abp1-carrying endocytic vesicles are pinched off from the plasma membrane within $10 \mathrm{~s}$ after its recruitment. Then, Abp1 departs from the post-internalized vesicles for re-use (Kaksonen et al. 2003; Kukulski et al. 2012). Abp1 is known to be an activator of the Arp2/3 complex that alone weakly nucleates actin assembly (Goode et al. 2001). However, it has been postulated that Abp1 helps Arp2/ 3 complex to be recruited to the actin filaments, thereby promoting the nucleation activity of Arp $2 / 3$ complex (Quintero-Monzon et al. 2005). Unlike mammalian cells, where chemical disruption of actin assembly only partially inhibits endocytosis (Lunn et al. 2000; Moskowitz et al. 2003), actin polymerization in yeast has been shown to be essential for endocytosis, based on the fact that cells treated with Latrunculin A exhibited no endocytic invagination 

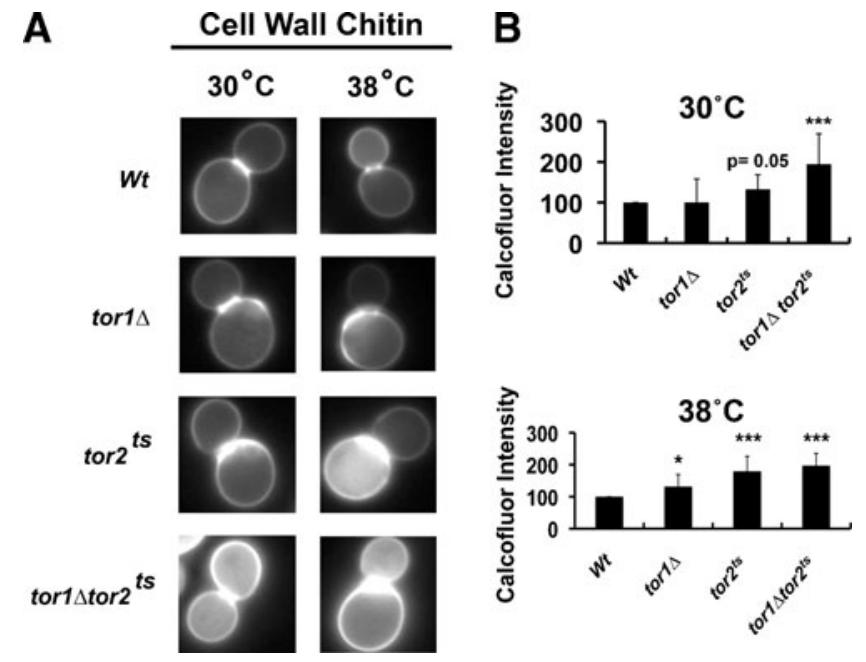

Figure 4. tor mutant cells have increase amount of cell wall chitin. (A) Wt (KKY 0343), torls (KKY 0917), tor $2^{\text {ts }}$ (KKY 0918), tor $1 \Delta$ tor $2^{\text {ts }}$ (KKY 0919) cells were stained with calcofluor white to visualize chitin under the confocal microscopy at different temperatures. (B) The mean fluorescence intensity of calcofluor white per cell $(n=10)$. An asterisk $(*)$ indicates statistically significant difference $(\mathrm{p}<0.05)$ between the calcofluor mean intensity in Wt and each tor mutant. A triple asterisk $(* * *)$ indicates statistically significant difference, where $\mathrm{p}<0.0001$.

(Kukulski et al. 2012). Interestingly, as shown in figure 1F, it was obvious that less Abp1 protein was found at endocytic sites in all tor mutant cells (tor $1 \Delta$, tor $2^{\text {ts }}$ and tor $1 \Delta$ tor $\left.2^{\text {ts }}\right)$. In particular, in those mutants we observed nearly $70-80 \%$ reduction in fluorescence intensity of Abp1-GFP at endocytic patches that showed endocytic failure, pointing to a correlation between the amount of Abp1 with the scission success of endocytic patches in those cells. Nevertheless, it is not plausible to assert that the observed defects are simply attributed to the reduced amount of Abp1 at the endocytic site due to the fact that cells deficient in Abp1 exhibited no severe endocytic defects (Galletta et al. 2008). Rather, in the case that the total amount of the Arp2/ 3 activator Abp1 is less than the threshold level, one can expect that other functionally redundant Arp2/3 activators compensate for the partial loss of Abp1, possibly inducing the final step of the endocytic event, namely, the pinchingoff process. However, knowing that Abp1 physically interacts with many endocytic factors, including Sla1 (endocytic adaptor), Rvs167 (amphiphysin), and actinregulating kinases Ark1 and Prk1 (Colwill et al. 1999; Cope et al. 1999; Lila and Drubin 1997; Warren et al. 2002), it is more reasonable to propose that Abp1 in general plays a significant role in serving as an organizer of efficient endocytic machinery at the endocytic site. In this view, it is highly likely that tor mutant cells exhibit aberration in recruitment of key endocytic proteins (for example, Rvs167 and Sj12, see below), which may result in building a functionally less competent endocytic machinery, and thereby conferring low scission success (figures 1 and 2). Given that, in addition to its overlapping function with Tor1, Tor2 is uniquely implicated in organization of the actin cytoskeleton (Schmidt et al. 1996), it is surprising to observe a similar scission defect in tor $1 \Delta$ cells comparable to that of $\operatorname{tor} 2^{\text {ts }}$ cells. An aberration in actin organization in yeast is tightly correlated with endocytic defect, which is the case for tor $2^{\text {ts }}$ cells (Schmidt et al. 1996). Because no actin organization defect in torl mutant cells has been reported (Schmidt et al. 1996), our explanation for the observed scission defect in the background of tor $1 \Delta$ would be an indirect effect of the loss of TOR1. Nonetheless, the question of how a lesser amount of Abp1 is recruited to endocytic sites in tor mutant cells remains elusive. Unexpectedly we observed a significant increase in Abp1-GFP lifespan upon Tor2 overexpression (figure 1B and C). Our explanation for this phenotype would be that Tor2 overexpression causes a dominant negative effect on Abp1-GFP dynamics at the endocytic site. This effect could be attributed to one or both of the following conditions. Given Tor2 acts as a potent protein kinase, the overexpression of Tor2 may result in phosphorylation of endocytic factors non-specifically, which affects endocytic protein maturation. Alternatively, it may be that Tor2 interacts with endocytic factors non-specifically due to the excessive amount of Tor2, and thereby sequestering them not to be readily recruited to the endocytic site.

\subsection{Drastic reduction of Rvs167-GFP carrying endocytic sites in tor mutant cells}

Yeast genome contains the amphiphysins homologs, Rvs161 and Rvs167, which form a heterodimer, playing an important role in endocytosis (Douglas et al. 2009; Ren et al. 2006). Endocytic function of Rvs167 resides in its BAR domain since expression of BAR domain alone in rvs $167 \Delta$ cells leads to the restoration of functional endocytosis (Sivadon et al. 1997). Rvs167 is recruited to endocytic sites 2-3 s after Abp1 has been recruited (Kaksonen et al. 2005), and the targeting appears to be mediated via its BAR domain, which contain positive amino acid residues that bind to the anionic plasma membrane (Peter et al. 2004). Due to the fact that purified BAR domain proteins are able to induce membrane curvature or tubulation in vitro and that a significant fraction of endocytic patches in rvs $167 \Delta$ cells exhibits a 'retraction phenotype', Rvs167 was thought to promote endocytic scission (Peter et al. 2004; Kaksonen et al. 2005; Takei et al. 1999). But this idea is currently being challenged by a recent report which suggests Rvs167 is not essentially required for endocytic scission based on electron tomography results that 
most of invaginated endocytic pits undergo pinching-off process in the absence of Rvs167 (Kukulski et al. 2012). Rather, the study showed that Rvs167 is recruited to newlyformed shallow invaginations, determined to be $\sim 50 \mathrm{~nm}$ deep in length and after its recruitment further invagination (up to $100 \mathrm{~nm}$ ) occurs, concluding that Rvs167 functions in stimulating membrane tubulation. This important finding may provide a plausible explanation for our observations in this report; the inefficient recruitment of Rvs167-GFP (supplementary figure 1B) and reduced scission in tor mutant cells (figure 2). As stated earlier, Arp2/3 complex-mediated actin assembly stimulated by its activators including Abp1 at endocytic sites is essential for formation of invaginating pits. Due to a significant loss in the amount of Abp1 and a potential subsequent defect in forming functional endocytic machinery, we, to put it simply, speculate that the invaginated pit in tor mutant cells, especially in tor $2^{\text {ts }}$ and tor $1 \Delta$ tor $2^{\text {ts }}$ cells, might be shorter $(<50 \mathrm{~nm})$ on average than in Wt cells. As a result, Rvs167 could not readily be recruited to those shallow invaginations of the plasma membrane, and in turn further induction of invagination may be ceased. The complete dislocation of Rvs167-GFP into the cytoplasm in the double mutant (tor $1 \Delta$ tor $2^{\text {ts }}$ ) cells (figure 2 and supplementary figure 1B) may then arise from a condition where the vast majority of endocytic invaginations in the double mutant cells are too short in depth to be recognized by Rvs167.

\subsection{Tor mutants exhibited a drastic increase in $\mathrm{PIP}_{2}$ levels and significant chitin accumulation}

Sj12-GFP, which normally localizes to endocytic sites, was completely mislocalized to the cytoplasm of the tor double mutant cells (figure 3 and supplementary figure 1C), as is the case for Rvs167-GFP. Sj12 is a yeast synaptojanin-like proteins that hydrolyses phosphates of inositol ring of $\mathrm{PIP}_{2}$ to form PI (phosphoinositide), and yeast genome contains two more Sj12 homologs, Sj11 and Sj13 (Srinivasan et al. 1997; Stolz et al. 1998; Guo et al. 1999). Previously, a group of researchers reported that loss of $\mathrm{Sj} 12$ leads to its cytoplasmic homolog Sj11 to be recruited to endocytic sites to compensate for the loss of Sj12, concluding that both proteins share the same enzymatic activity (Sun et al. 2007). In light of finding that the severe mislocalization of Sj12 in the tor double mutant cells (figure 3), we investigated the localization of Sj11 and found that the GFP-fused-Sj11 is mainly located in the cytoplasm (data not shown). As such, we reasoned that membrane $\mathrm{PIP}_{2}$ levels in the double mutant should increase due to both $\mathrm{Sj} 11$ and $\mathrm{Sj} 12$ not being detected at the membrane cortex. Indeed, according to our GFP2XPH fluorescence intensity results (figure 3 ), there was a drastic elevation in $\mathrm{PIP}_{2}$ level in all tor mutant cells (tor $1 \Delta$, $\operatorname{tor} 2^{t s}$, and tor $1 \Delta$ tor $2^{t s}$ ) compared to that in $\mathrm{Wt}$ cells.
Inappropriate balance in membrane $\mathrm{PIP}_{2}$ levels can adversely affect endocytosis. Since many actin-binding endocytic proteins are known to physically interact with $\mathrm{PIP}_{2}$ at endocytic sites, low levels of $\mathrm{PIP}_{2}$ result in an actin assembly defect, accompanied by a significant blocking of endocytosis (Abe et al. 2008; Logan and Mandato 2006). High levels of $\mathrm{PIP}_{2}$ are in general associated with hyperstimulation of actin assembly, which also causes endocytic deficiency (Sun et al. 2007). Intriguingly, the observed hyperaccumulation of $\mathrm{PIP}_{2}$ appears not to be correlated with less recruitment of endocytic proteins (Abp1 and Rvs167) in our tor mutant cells (figures 1 and 2). We, at the moment, are not able to adequately explain the molecular basis of this discrepancy. However, given the fact that $\mathrm{PIP}_{2}$ binds and activates the Rom2-Rho1 pathway that controls endocytosis, we speculate that abnormally high accumulation of $\mathrm{PIP}_{2}$ in tor mutant cells may dysregulate the Rom2-mediated endocytosis. The yeast cell wall comprised mostly of chitin is implicated in protecting the cell from rapid changes in osmotic pressure and against environmental stresses. Upon sensing of cell wall weakening (or damage), yeast cells up-regulate chitin synthesis to stabilize the cell wall (Dallies et al. 1998). In conditions where the mutant Tor2 protein is partially and completely inactivated, we found that cell wall chitin levels are significantly increased up to $100 \%$ (figure 4). Given the fact that an endocytic blockade impairs internalization of Chs3, the chitin synthase subunit, leading to a significant increase in chitin synthesis (Reyes et al. 2007), it is plausible to postulate that the drastic increase in chitin levels in the tor mutant $\left(\right.$ tor $2^{t s}$ and tor $1 \Delta$ tor $2^{t s}$ ) might be due to an incompetent endocytosis, at least, of Chs3.

\section{Acknowledgements}

We thank Dr Hall (University of Basel) for providing tor mutant strains (tor $1 \Delta$, tor $2^{\text {ts }}$, tor $1 \Delta$ tor $2^{t s}$, and tor 2 overexpressing mutant). This work was supported by a National Scientific Foundation Grant 0923024 (to K KIM) and by thesis funding from Missouri State University (B Tenay).

\section{References}

Abe N, Inoue T, Galvez T, Klein L and Meyer T 2008 Dissecting the role of PtdIns(4,5)P2 in endocytosis and recycling of the transferrin receptor. J. Cell Sci. 121 1488-1494

Aronova S, Wedaman K, Anderson S, Yates J, 3rd, and Powers T 2007 Probing the membrane environment of the TOR kinases reveals functional interactions between TORC1, actin, and membrane trafficking in Saccharomyces cerevisiae. Mol. Biol. Cell 18 2779-2794

Balguerie A, Sivadon P, Bonneu M and Aigle M 1999 Rvs167p, the budding yeast homolog of amphiphysin, colocalizes with actin patches. J. Cell Sci. 112 2529-2537 
Barbet NC, Schneider U, Helliwell SB, Stansfield I, Tuite MF and Hall MN 1996 TOR controls translation initiation and early G1 progression in yeast. Mol. Biol. Cell 7 25-42

Berchtold D and Walther TC 2009 TORC2 plasma membrane localization is essential for cell viability and restricted to a distinct domain. Mol. Biol. Cell 20 1565-1575

Bickle M, Delley PA, Schmidt A and Hall MN 1998 Cell wall integrity modulates RHO1 activity via the exchange factor ROM2. EMBO J. 17 2235-2245

Cafferkey R, Young PR, McLaughlin MM, Bergsma DJ, Koltin Y, Sathe GM, Faucette L, Eng WK, Johnson RK and Livi GP 1993 Dominant missense mutations in a novel yeast protein related to mammalian phosphatidylinositol 3-kinase and VPS34 abrogate rapamycin cytotoxicity. Mol. Cell. Biol. 13 6012-6023

Cardenas ME and Heitman J 1995 FKBP12-rapamycin target TOR2 is a vacuolar protein with an associated phosphatidylinositol-4 kinase activity. EMBO J. 14 5892-5907

Chen DC, Yang BC and Kuo TT 1992 One-step transformation of yeast in stationary phase. Curr. Genet. 21 83-84

Colwill K, Field D, Moore L, Friesen J and Andrews B 1999 In vivo analysis of the domains of yeast Rvs167p suggests Rvs167p function is mediated through multiple protein interactions. Genetics 152 881-893

Cope MJ, Yang S, Shang C and Drubin DG 1999 Novel protein kinases Ark1p and Prk1p associate with and regulate the cortical actin cytoskeleton in budding yeast. J. Cell Biol. 144 1203-1218

Dallies N, Francois J and Paquet V 1998 A new method for quantitative determination of polysaccharides in the yeast cell wall. Application to the cell wall defective mutants of Saccharomyces cerevisiae. Yeast 14 1297-1306

deHart AK, Schnell JD, Allen DA, Tsai JY and Hicke L 2003 Receptor internalization in yeast requires the Tor2-Rho1 signaling pathway. Mol. Biol. Cell 14 4676-4684

Douglas LM, Martin SW and Konopka JB 2009 BAR domain proteins Rvs161 and Rvs167 contribute to Candida albicans endocytosis, morphogenesis, and virulence. Infect. Immun. 77 $4150-4160$

Engqvist-Goldstein AE and Drubin DG 2003 Actin assembly and endocytosis: from yeast to mammals. Annu. Rev. Cell Dev. Biol. 19 287-332

Galletta BJ, Chuang DY and Cooper JA 2008 Distinct roles for Arp2/3 regulators in actin assembly and endocytosis. PLoS Biol. 6e1

Goode BL, Rodal AA, Barnes G and Drubin DG 2001 Activation of the Arp2/3 complex by the actin filament binding protein Abp1p. J. Cell Biol. 153 627-634

Guo S, Stolz LE, Lemrow SM and York JD 1999 SAC1-like domains of yeast SAC1, INP52, and INP53 and of human synaptojanin encode polyphosphoinositide phosphatases. $J$. Biol. Chem. 274 12990-12995

Hall MN 2008 mTOR-what does it do? Transplant. Proc. 40 S5-S8

Helliwell SB, Wagner P, Kunz J, Deuter-Reinhard M, Henriquez R and Hall MN 1994 TOR1 and TOR2 are structurally and functionally similar but not identical phosphatidylinositol kinase homologues in yeast. Mol. Biol. Cell 5 105-118

Idrissi FZ, Grotsch H, Fernandez-Golbano IM, Presciatto-Baschong C, Riezman H and Geli MI 2008 Distinct acto/myosin-I structures associate with endocytic profiles at the plasma membrane. J. Cell Biol. 180 1219-1232

Jacinto E, Loewith R, Schmidt A, Lin S, Ruegg MA, Hall A and Hall MN 2004 Mammalian TOR complex 2 controls the actin cytoskeleton and is rapamycin insensitive. Nat. Cell Biol. 6 1122-1128

Kaksonen M, Sun Y and Drubin DG 2003 A pathway for association of receptors, adaptors, and actin during endocytic internalization. Cell 115 475-487

Kaksonen M, Toret CP and Drubin DG 2005 A modular design for the clathrin- and actin-mediated endocytosis machinery. Cell 123 305-320

Kim DH, Sarbassov DD, Ali SM, King JE, Latek RR, ErdjumentBromage H, Tempst P and Sabatini DM 2002 mTOR interacts with raptor to form a nutrient-sensitive complex that signals to the cell growth machinery. Cell 110 163-175

Kim K, Galletta BJ, Schmidt KO, Chang FS, Blumer KJ and Cooper JA 2006 Actin-based motility during endocytosis in budding yeast. Mol. Biol. Cell 17 1354-1363

Kukulski W, Schorb M, Kaksonen M and Briggs JA 2012 Plasma membrane reshaping during endocytosis is revealed by timeresolved electron tomography. Cell 150 508-520

Kunz J, Henriquez R, Schneider U, Deuter-Reinhard M, Movva NR and Hall MN 1993 Target of rapamycin in yeast, TOR2, is an essential phosphatidylinositol kinase homolog required for G1 progression. Cell 73 585-596

Laplante M and Sabatini DM 2012 mTOR signaling in growth control and disease. Cell 149 274-293

Lila T and Drubin DG 1997 Evidence for physical and functional interactions among two Saccharomyces cerevisiae $\mathrm{SH} 3$ domain proteins, an adenylyl cyclase-associated protein and the actin cytoskeleton. Mol. Biol. Cell 8 367-385

Loewith R, Jacinto E, Wullschleger S, Lorberg A, Crespo JL, Bonenfant D, Oppliger W, Jenoe P and Hall MN 2002 Two TOR complexes, only one of which is rapamycin sensitive, have distinct roles in cell growth control. Mol. Cell 10 457-468

Logan MR and Mandato CA 2006 Regulation of the actin cytoskeleton by PIP2 in cytokinesis. Biol. Cell 98 377-388

Longtine MS, McKenzie A, 3rd, Demarini DJ, Shah NG, Wach A, Brachat A, Philippsen P and Pringle JR 1998 Additional modules for versatile and economical PCR-based gene deletion and modification in Saccharomyces cerevisiae. Yeast 14 953-961

Lunn JA, Wong H, Rozengurt E and Walsh JH 2000 Requirement of cortical actin organization for bombesin, endothelin, and EGF receptor internalization. Am. J. Physiol. Cell Physiol. 279 C2019-C2027

Manning BD and Cantley LC 2007 AKT/PKB signaling: navigating downstream. Cell 129 1261-1274

Moskowitz HS, Heuser J, McGraw TE and Ryan TA 2003 Targeted chemical disruption of clathrin function in living cells. Mol. Biol. Cell 14 4437-4447

Murphy ER, Boxberger J, Colvin R, Lee SJ, Zahn G, Loor F and Kim K 2011 Pill, an eisosome organizer, plays an important role in the recruitment of synaptojanins and amphiphysins to facilitate receptor-mediated endocytosis in yeast. Eur. J. Cell Biol. 90 825-833

Nannapaneni S, Wang D, Jain S, Schroeder B, Highfill C, Reustle L, Pittsley D, Maysent A, Moulder S, McDowell R, et al. 2010 
The yeast dynamin-like protein Vps1:vps1 mutations perturb the internalization and the motility of endocytic vesicles and endosomes via disorganization of the actin cytoskeleton. Eur. J. Cell Biol. 89 499-508

Newpher TM, Smith RP, Lemmon V and Lemmon SK 2005 In vivo dynamics of clathrin and its adaptor-dependent recruitment to the actin-based endocytic machinery in yeast. Dev. Cell 9 87-98

Peter BJ, Kent HM, Mills IG, Vallis Y, Butler PJ, Evans PR and McMahon HT 2004 BAR domains as sensors of membrane curvature: the amphiphysin BAR structure. Science $\mathbf{3 0 3}$ 495-499

Quintero-Monzon O, Rodal AA, Strokopytov B, Almo SC and Goode BL 2005 Structural and functional dissection of the Abp1 ADFH actin-binding domain reveals versatile in vivo adapter functions. Mol. Biol. Cell 16 3128-3139

Ren G, Vajjhala P, Lee JS, Winsor B and Munn AL 2006 The BAR domain proteins: molding membranes in fission, fusion, and phagy. Microbiol. Mol. Biol. Rev. 70 37-120

Reyes A, Sanz M, Duran A and Roncero C 2007 Chitin synthase III requires Chs $4 p$-dependent translocation of $\mathrm{Chs} 3 \mathrm{p}$ into the plasma membrane. J. Cell Sci. 120 1998-2009

Schmidt A, Kunz J and Hall MN 1996 TOR2 is required for organization of the actin cytoskeleton in yeast. Proc. Nat. Acad. Sci. USA 93 13780-13785

Sivadon P, Crouzet M and Aigle M 1997 Functional assessment of the yeast Rvs161 and Rvs167 protein domains. FEBS Lett. 417 $21-27$

Smaczynska-de R II, Allwood EG, Mishra R, Booth WI, Aghamohammadzadeh S, Goldberg MW and Ayscough KR 2012 Yeast dynamin Vps1 and amphiphysin Rvs167 function together during endocytosis. Traffic 13 317-328

Srinivasan S, Seaman M, Nemoto Y, Daniell L, Suchy SF, Emr S, De Camilli P and Nussbaum R 1997 Disruption of three phosphatidylinositol-polyphosphate 5-phosphatase genes from Saccharomyces cerevisiae results in pleiotropic abnormalities of vacuole morphology, cell shape, and osmohomeostasis. Eur. J. Cell Biol. 74 350-360

Stefan CJ, Audhya A and Emr SD 2002 The yeast synaptojanin-like proteins control the cellular distribution of phosphatidylinositol (4,5)-bisphosphate. Mol. Biol. Cell 13 542-557

Stefan CJ, Padilla SM, Audhya A and Emr SD 2005 The phosphoinositide phosphatase Sj12 is recruited to cortical actin patches in the control of vesicle formation and fission during endocytosis. Mol. Cell. Biol. 25 2910-2923

Stolz LE, Huynh CV, Thorner J and York JD 1998 Identification and characterization of an essential family of inositol polyphosphate 5-phosphatases (INP51, INP52 and INP53 gene products) in the yeast Saccharomyces cerevisiae. Genetics 148 1715-1729

Sturgill TW, Cohen A, Diefenbacher M, Trautwein M, Martin DE and Hall MN 2008 TOR1 and TOR2 have distinct locations in live cells. Eukaryotic Cell 7 1819-1830

Sun Y, Carroll S, Kaksonen M, Toshima JY and Drubin DG 2007 PtdIns(4,5)P2 turnover is required for multiple stages during clathrin- and actin-dependent endocytic internalization. J. Cell Biol. 177 355-367

Takei K, Slepnev VI, Haucke V and De Camilli P 1999 Functional partnership between amphiphysin and dynamin in clathrinmediated endocytosis. Nat. Cell Biol. $133-39$

Toret CP, Lee L, Sekiya-Kawasaki M and Drubin DG 2008 Multiple pathways regulate endocytic coat disassembly in Saccharomyces cerevisiae for optimal downstream trafficking. Traffic 9 848-859

Warren DT, Andrews PD, Gourlay CW and Ayscough KR 2002 Sla1p couples the yeast endocytic machinery to proteins regulating actin dynamics. J. Cell Sci. 115 1703-1715

Wedaman KP, Reinke A, Anderson S, Yates J, 3rd, McCaffery JM and Powers T 2003 Tor kinases are in distinct membraneassociated protein complexes in Saccharomyces cerevisiae. Mol. Biol. Cell 14 1204-1220

MS received 16 January 2013; accepted 15 March 2013

Corresponding editor: VeEnA K PARnAIK 